\title{
Exploring User Motor Behaviour in Bimanual Interactive Video Games
}

\author{
N. Pena Perez ${ }^{*}$, L. Tokarchuk ${ }^{\dagger}$, E. Burdet ${ }^{\ddagger}$, I. Farkhatdinov ${ }^{\S}$ \\ School of Electronic Engineering and Computer Science, Queen Mary University London, UK ${ }^{* \dagger \S}$ \\ Department of Bioengineering, Imperial College of Science, Medicine and Technology, London, UK $* \ddagger$ \\ \{n.penaperez,laurissa.tokarchuk,i.farkhatdinov\}@qmul.ac.uk*†§, e.burdet@imperial.ac.uk ${ }^{\ddagger}$
}

\begin{abstract}
Video games have proved very valuable in rehabilitation technologies. They guide therapy and keep patients engaged and motivated. However, in order to realize their full potential, a good understanding is required of the players' motor control. In particular, little is known regarding player behaviour in tasks demanding bimanual interaction. In this work, an experiment was designed to improve the understanding of such tasks. A driving game was developed in which players were asked to guide a differential wheeled robot (depicted as a rocket) along a trajectory. The rocket could be manipulated by using an Xbox controller's triggers, each supplying torque to the corresponding side of the robot. Such a task is redundant, i.e. there exists an infinite number of input combinations to yield a given outcome. This allows the player to strategize according to their own preference. 10 participants were recruited to play this game and their input data was logged for subsequent analysis. Two different motor strategies were identified: an "intermittent" input pattern versus a "continuous" one. It is hypothesized that the choice of behaviour depends on motor skill and minimization of effort and error. Further testing is necessary to determine the exact relationship between these aspects.
\end{abstract}

Index Terms-Video Games; Rehabilitation; Bimanual Coordination; Motor Behaviour.

\section{INTRODUCTION}

Stroke survivors suffer from serious long term motor impairments that compromise their ability to perform activities of daily living (ADLs) and to communicate with their environment. Rehabilitation is essential to return basic sensing and motor abilities to the limbs. However, training is repetitive, exhausting and complex to practise without supervision, which affects patients' motivation and psychological well-being and limits the therapy to clinic appointments [1]-[3].

In this context video games have been proposed as a promising tool for improving current stroke rehabilitation therapies. They offer an environment where exercises can be performed in a more motivating and engaging manner [4], [5]. Outside the clinic, games can encourage patients to perform more repetitions of simple exercises while storing relevant data that allows therapist to follow up recovery [6], [7]. An extended advantage of video games is that they allow representation of several inputs, enabling bimanual and/or social training modes.

The importance of bimanual training arises from the fact that many ADLs require that the two hands perform simultaneously when interacting with a single object, such as opening a bottle or fastening buttons.

978-1-7281-1884-0/19/\$31.00 @2019 IEEE
These tasks require a high level of coordination and the exertion of adequate forces. Therefore a growing number of studies have investigated motor coordination [8] and the efficacy of bilateral training after stroke [9]. This could contribute to improving the patients' quality of life by allowing them to be more independent in the practice of ADLs since training bimanual movements may help restoring coordination patterns altered after suffering a stroke [10]. In addition, recovery in hemiparetic patients may be enhanced trough interhemispheric communication through operation of the healthy arm [11].

Despite many theories contributing to explain the nature of coordination, there is a general agreement that it is highly task dependent [12], promoting different motor strategies during different tasks. Video games enjoy enormous flexibility. Due to this design flexibility, games provide a platform in which multiple different tasks can be considered. Therefore, a player's objective can be designed such that it promotes patterns of behaviour central to the player's re-acquiring of motor skills. However, task dynamics must be carefully designed to promote an adequate pattern.

A bimanual task can be characterized by its goal, its spatial and temporal symmetry and its coupling. Goal-based characterization of tasks depends on both hands pursuing the same objective or working towards different goals. Spatial and temporal symmetry based characterization is instead determined by how the hands move. Questions considered include: Do the hands perform the same movement? And do they move at the same rhythm? Finally, coupling based characterization depends on whether or not the hands are mechanically linked as it would happen during object manipulation.

Existing research has focused primarily on symmetric and dual-goal based characterizations [9], [13]. There is therefore a gap in the understanding of motor behaviour in single-goal, non-symmetric tasks. This work will examine what motor behaviours arise when participants are asked to perform a single-goal, non-symmetric task. To achieve this, a redundant bimanual task, in which both hands need to actively collaborate to succeed has been designed.

It is fundamental to understand how movement is coordinated when both hands are forced to collaborate for the same objective. In rehabilitation, this would prevent participants from adopting a slacking behaviour, which may arise when one of the hands is weakened and task dynamics do not 
TABLE I

RELEVANT PARTICIPANT FEATURES.

\begin{tabular}{|c|c|c|c|c|}
\hline $\begin{array}{c}\text { Player } \\
\text { ID }\end{array}$ & Group & Handedness* & $\begin{array}{c}\text { Game } \\
\text { Experience }\end{array}$ & $\begin{array}{c}\text { Controller } \\
\text { Experience** }\end{array}$ \\
\hline A & Left & 66.66 & Monthly & None \\
\hline B & Left & 62.5 & Weekly & Medium \\
\hline C & Left & -5.8 & Daily & None \\
\hline D & Left & 100 & Daily & Medium \\
\hline E & Left & -6.66 & Weekly & None \\
\hline F & Left & 87.5 & Weekly & Medium \\
\hline G & Right & 100 & Monthly & Advanced \\
\hline H & Right & 76.47 & Weekly & Medium \\
\hline I & Right & 87.5 & Weekly & Medium \\
\hline J & Right & 100 & Weekly & Expert \\
\hline
\end{tabular}

* The Edinburgh Handedness Inventory produces a score between -100 to 100 . Participants who get a negative score classify as lefthanded, and those with a positive score, classify as right-handed. Participants with scores between -70 and 70 are additionally considered inconsistent-handed [15].

*** Presents participant expertise with Xbox 360 controllers.

enforce hand collaboration. However, the fact that the task is not enforcing symmetry and is redundant allows for hands to be controlled differently. In this way, if one of the hands was not as skilled as the other there would still be a coordination strategy such that the skilled hand would be compensating for the mistakes of the other. Mace et al. [14] explored this type of assistance during dyadic coordination. The bimanual implementation can potentially aid hemiparetic patients during rehabilitation. However, to apply this type of video game for rehabilitation purposes, we first need to understand which motor behaviours arise naturally when hands are coordinating in this task.

This study will therefore investigate bilateral coordination, in particular how the left and right index fingers must coordinate to control a virtual object. In the proposed task there are no forced spatial or temporal symmetries determined by the task dynamics and left and right fingers are only coupled trough visual feedback. Because of the task's redundancy, arising patterns would only be defined by player preference and skill. However, activity from both hands is necessary to achieve the goal.

\section{Methods}

\section{A. Participants}

Ten young adults (mean age: 23.9 $\pm 2.77,1$ female and 9 males), volunteered for the study. None of them reported any mental, cognitive, or other neurological disorder, or suffered from any motor or visual injury. Participants were given an information sheet containing all the required information about the study, task instructions and data handling. The experiment was approved by the local Ethics Committee at the University of York and informed consent was collected prior to participating in the study. Participants were all recruited from the University community, and all of them had previous experience with video games at different levels. Their handedness was verified using the Edinburgh Handedness Inventory (Table I) [15].

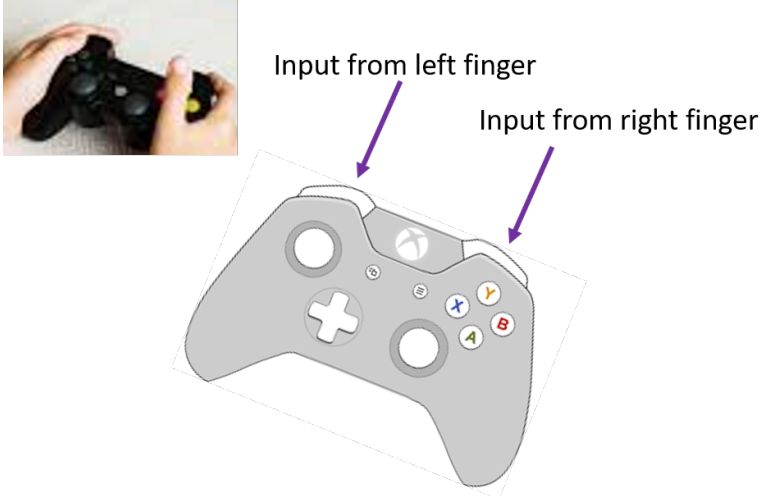

Fig. 1. Schematics of the Xbox 360 controller used for this experiment and how participants were holding it.

\section{B. Materials}

1) Game: A top-down perspective driving game was developed in Unity. A rocket was controlled by pressing the left and right triggers of an Xbox 360 controller (Fig. 1). Increasing the pressure of one trigger caused the rocket to turn in that direction, while keeping both triggers pressed with the same intensity kept the rocket travelling on a straight line. The game consisted of one single scene, with a static camera, allowing players to see components at all times. Graphics were simple to minimize possible distractions. The scene had three main components: a rocket as the object to be controlled, a road or path to be followed and an arrow indicating the first turning direction (Figure 2). Each round of game play required driving the rocket from the start to the finish line, driving along the circles in the order indicated by the arrow. Participants were asked to keep the rocket to the center of the road, and were told to focus on their accuracy without regard to the time taken to complete the task.

2) Building the redundant task: To ensure the task's redundancy a differential wheeled robot model was used to simulate the rocket dynamics [16]. The speed at which participants had to travel was not specified, so in order to follow the road path they were able to use infinitely many combinations of right wheel/left wheel torque inputs. The factor determining the shape of those inputs would be therefore only determined by the player's preference and strategy to achieve the goal.

The rocket dynamics were governed by two coupled second order differential equations (1) and (2) that relate a torque input to the right and left wheels $\left(\tau_{r}\right.$ and $\tau_{r}$, respectively) with their corresponding angular acceleration $\left(\frac{d \omega_{r}}{d t}, \frac{d \omega_{l}}{d t}\right)$ and velocity $\left(\omega_{r}, \omega_{r}\right)$. The input, used as torque for the wheels, is a mapping of the pressure that participants exerted on the triggers.

$$
\begin{gathered}
\tau_{r}=M_{r} \frac{d \omega_{r}}{d t}+N_{r} \frac{d \omega_{l}}{d t}+P_{r} \omega_{r} \\
\tau_{l}=M_{l} \frac{d \omega_{l}}{d t}+N_{l} \frac{d \omega_{r}}{d t}+P_{l} \omega_{l}
\end{gathered}
$$




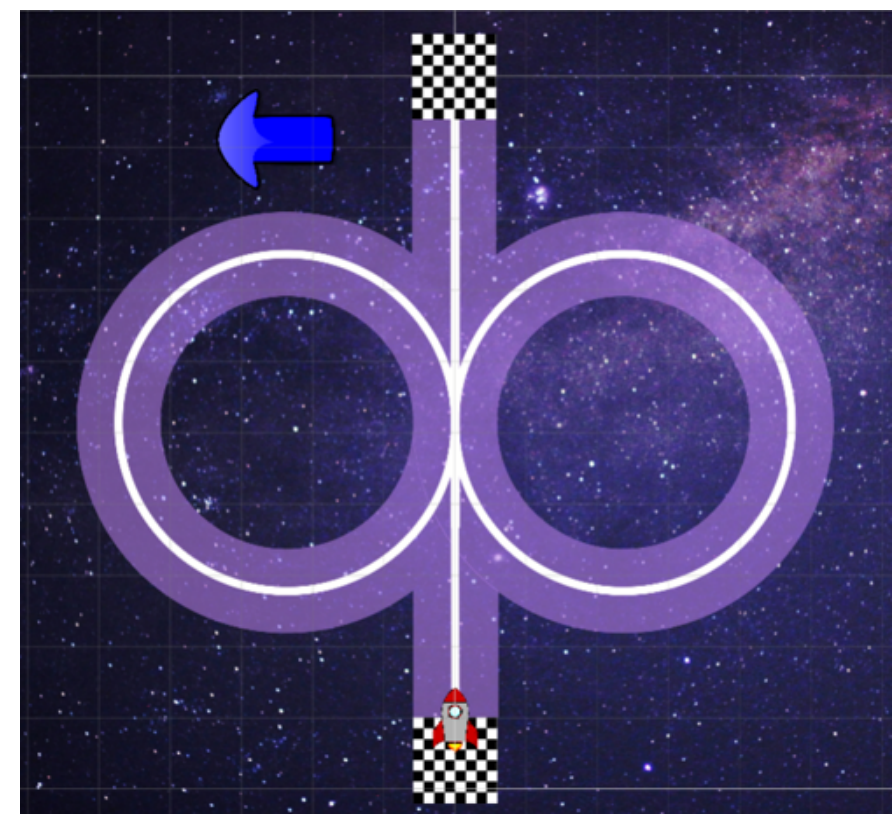

Fig. 2. Rocket game scene in Unity. The road was designed in Inkscape; the arrow, the rocket and the background were found online as public domain licensed graphics.

These equations were solved numerically for $\omega_{r}$ and $\omega_{l}$, and were used to compute linear, $v$, and angular velocities, $\omega$ of the rocket.

$$
\left(\begin{array}{c}
v \\
\omega
\end{array}\right)=\left(\begin{array}{ll}
\frac{R_{r}}{2} & \frac{R_{l}}{2} \\
\frac{R_{r}}{T} & \frac{R_{l}}{T}
\end{array}\right)\left(\begin{array}{l}
\omega_{l} \\
\omega_{r}
\end{array}\right)
$$

The description of the model can be found in the Appendix.

\section{Experimental Procedure}

Just before starting the experiment participants were reminded the task instructions. To play the game they were seated at a computer desk holding an Xbox 360 controller. The experiment consisted of two phases. In the first phase, participants were allowed to familiarize with the rocket's control in a short practice session of half a minute. During this practice, players could not see any road and the rocket could move freely in space. In the second phase, participants played ten rounds of the game, each of which had a different turning order than the previous. Participants were divided into two groups depending on which direction they turned first, left or right. The "Left" group was integrated by four right handed and two left handed participants, while the "Right" group was integrated by only right handed participants (Table I). The duration of the experiment varied among players, as they completed the task at different speeds (mean time per round: $54.88 \pm 22.07 \mathrm{~s}$ ). After finishing the experiment, participants were given a questionnaire, consisting of questions about demographics and their perceived performance and behaviour.

\section{Data Analysis}

Data was recorded during the second phase of the experiment and stored in text files. Six different variables were recorded:
- Left and right inputs, which are a mapping of the force that participants were exerting on the triggers that oscillated in a range of 0 (trigger released) to 1 (completely pressed). A multiplier of 2.5 was used in the game, to adequate response to road shape.

- Rocket movement, measured as the displacement in the $x$ and $y$ directions for each frame.

- Linear speed of the car.

- Time at which each variable was recorded since the trial started.

The recorded data was imported to MATLAB for processing and analysis. Processing involved remapping the recorded inputs to a range of 0 to 1 , to remove the effect of the multipliers, and calculating the cumulative positions from the displacement increments.

Analysis was divided into four main blocks. In the first place, recorded data was visually inspected in order to characterize the shape of the inputs and outputs and their variation among participants.

In the second place, the SPARC measure of smoothness [17] was chosen to analyze the motor behaviour of the participants. SPARC (4) is a dimensionless measure of the path length of the frequency spectrum of the signal profile over the bandwidth appropriate for the action. This metric was selected above other common smoothness measures in the motor control literature due to its robustness, sensitivity and validity outside discrete tasks. The smoothness of the recorded inputs was therefore assessed using this metric.

$$
S P A R C \triangleq \int_{0}^{\omega_{c}}\left[\left(\frac{1}{\omega_{c}}\right)^{2}+\left(\frac{d \hat{V}(\omega)}{\omega}\right)^{2}\right]^{\frac{1}{2}} d \omega
$$

with $\hat{V}(\omega)=\frac{V(\omega)}{V(0)}$, where $V(\omega)$ is the Fourier magnitude spectrum, $\hat{V}(\omega)$ is the normalized magnitude spectrum, $\mathrm{V}(0)$ is the DC magnitude and $\omega_{c}$ is defined as in (5).

$$
\omega_{c} \triangleq \min \left\{\omega_{c}^{m} a x, \min \{\omega, \hat{V}(r)<\bar{V} \forall r>\omega\}\right\}
$$

In the third place, the relationship between the input profiles and the output trajectories was studied in order to understand how different strategies affected performance. Performance was evaluated by computing the mean error (6) between the participant's trajectory and the reference along each single trial. The error was computed as the distance to the closest point in the reference curve for each time point of the subject's trajectory. Let $x_{r} \in X$ be the set of $\mathrm{x}$ coordinate points belonging to the reference curve and $y_{r} \in Y$ the set of y coordinate points belonging to the reference curve. Then the error was calculated as indicated by (6)

$$
\text { Error } \left.=\sum_{i=0}^{N}\left(\min _{x_{r} \in X, y_{r} \in Y} \sqrt{\left(x-x_{r}\right)^{2}+\left(y-y_{r}\right)^{2}}\right)\right) .
$$

The last block of analysis involved exploring the evolution of the different variables along trials. 

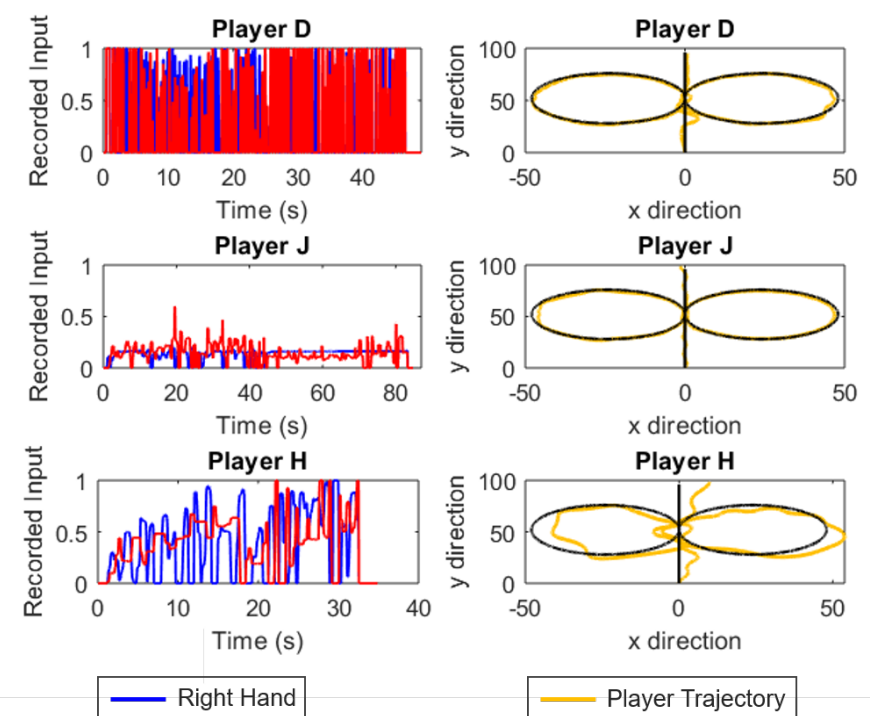

Left Hand

Fig. 3. Last trial's right hand and left hand inputs (Left), and corresponding output trajectories Right for players D, J and H. The input to the Xbox 360 controller maps to a range of 0 to 1 (no units) the pressure level exerted by the player on each trigger. The trajectory of each player (in yellow) is presented with the trajectory they were asked to follow (in black).

\section{RESULTS}

The recorded data was explored to understand if different motor behaviours had arisen. Processed data can be observed in Fig. 3. The recorded input along time and the trajectories for the last round of players D, J and $\mathrm{H}$ are presented. Looking at the trajectories, it seems that participants $\mathrm{D}$ and $\mathbf{J}$ were more accurate tracking the road than player $\mathrm{H}$. It can be observed in the input signals that players $\mathrm{D}$ and $\mathrm{H}$ completed the round in a shorter time than $\mathrm{J}$. In addition, the input signals vary among participants, both in amplitude and tendencies.

The motor behaviour of the participants can be further studied by exploring the recorded inputs. Fig. 4 shows the smoothness distributions for all rounds of each player. Values closer to zero indicate a smoother control. Smoothness of both left and right inputs are presented in the graph, although no obvious differences can be appreciated by visual inspection. However, clear air can be seen between the smoothness of participants $\mathrm{A}, \mathrm{D}$ and $\mathrm{F}$ with respect to the others.

To understand the possible causes determining a certain behaviour, the participants' trajectory and the time it took them to complete each trial were also considered. Fig. 5 shows how performance varies as a function of the smoothness.

Finally, smoothness changes along trials was explored. However, only participant D was observed to show a clear change in behaviour. Trials $1,2.3$ and 10 are represented in Fig. 6 to illustrate this behaviour.

\section{Discussion}

The results obtained indicate the existence of different motor behaviours based on different input tendencies and time

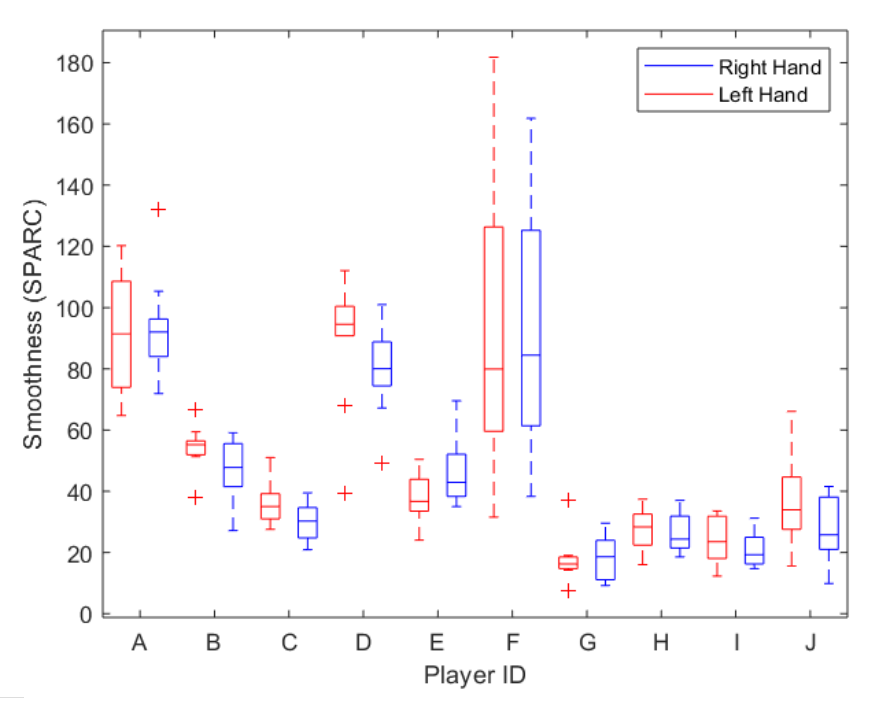

Fig. 4. Smoothness for each player along every trial. Smoothness is calculated as one value per trial, the closest to zero the more smooth the input was. Each red box includes the smoothness of the left hand for the ten trials of that particular participant. Boxes in blue, present the right hand smoothness.

spent. Fig. 3 shows recorded inputs (triggers) and outputs (trajectory) for the last round of a selection of three players. After 10 rounds of game play, their motor behaviours differ significantly. Players D and J obtain similar trajectories (very accurate in tracking) with completely different input tendencies. Player D pushed the triggers to their maximum in a discontinuous fashion, while player $\mathrm{J}$ maintained a soft, constant pressure during the entire round (particularly in their right hand). Player $\mathrm{H}$, however, obtained a less accurate result during the last trial. This participant seems to have provided an input more similar in shape to that of player $\mathrm{J}$, but higher in magnitude and taking half of the time.

It could be hypothesized that after more trials player $\mathrm{H}$ could have learned to modulate their actions to improve the accuracy of the trajectory. A tracking improvement could presumably be related to either a change to a more suitable control input or by increasing the time spent in the task. In order to explore if this hypothesis is sensitive, first the motor input was characterized using the SPARC metric. This was followed by an analysis of the relationship between performance, motor behaviour and time spent in the task.

In Fig. 4, participants A, D and F stand out due to their poor smoothness. This indicates a motor behaviour that involved intermittently pressing the controller, manipulating them as if they were switches. The other players, however, have supplied a more constant input, carefully regulating the pressure level on the triggers. It was therefore hypothesized that participants chose between two different types of behaviours, the "intermittent" and the "continuous". In general, participants seemed to be aware of their behaviour as shown by the data collected in the questionnaire. When asked about their perceived strategy player A said to "use shorter presses", player D to "move slowly, in bursts of speed" and player F to press the controllers 

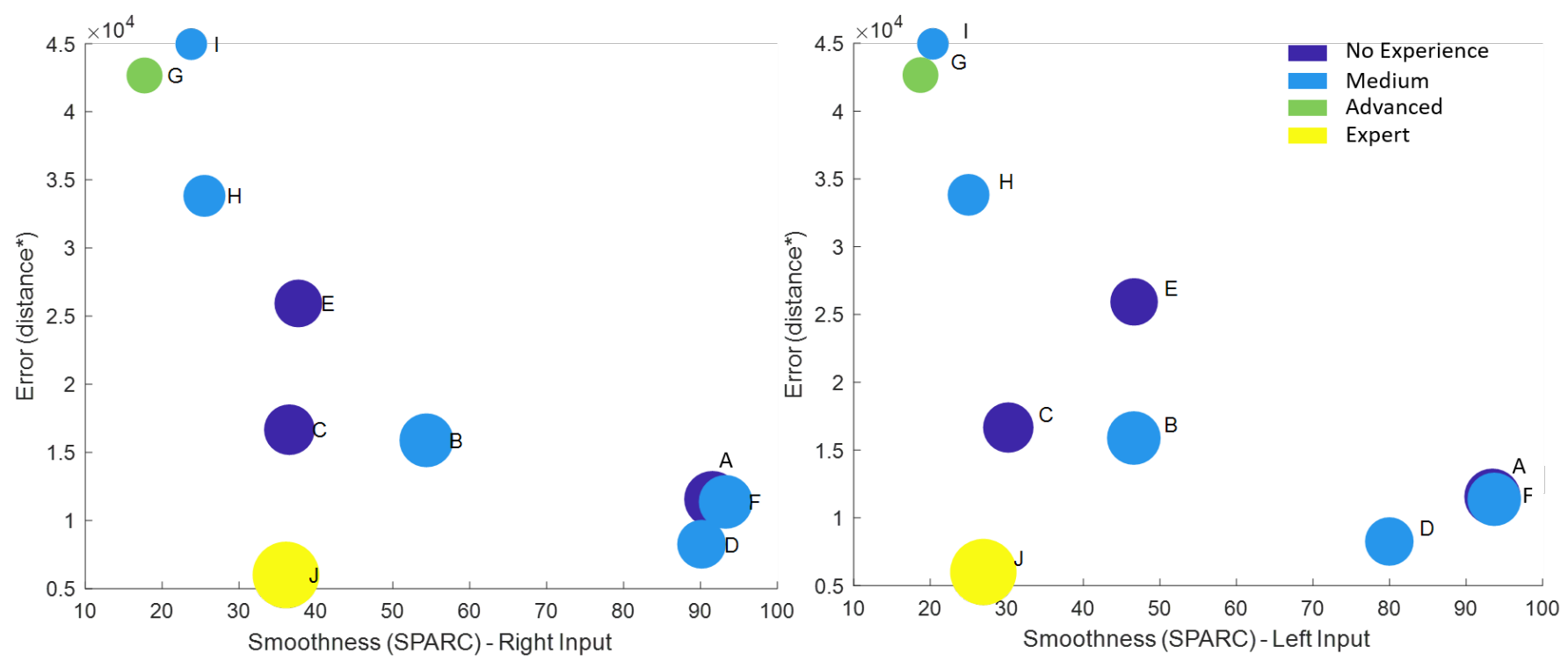

Fig. 5. Error versus smoothness. The error was computed as the distance to the closest point in the reference curve for each time point of the subject's trajectory, averaged over trials. The area of the data points shows the average time a participant to complete the trials (the more time the largest). Participant data points are labelled according to their identifiers and their experience with the used controllers is color coded. *Error units correspond to virtual distance units.

"individually intermittently". Other participants mentioned to have traveled "slow and steady" or "smooth and accurate". This indicates some level of awareness on the behaviour chosen.

Fig. 5 shows that participants that used the 'intermittent' behaviour obtained in general a low error, implying good tracking performance. However, for participants using the 'continuous' behaviour the error seems to depend on both their experience using Xbox 360 controllers and the time they spent in the task.

It has been proposed that humans adapt to new dynamic environments by minimizing a cost function that weighs error and effort [18]. In this study, the main motivation is expected to be a reduction of the error, i.e. keeping close to the center of the road. On the one hand, the 'continuous' behaviour seems to convey less effort, and is more optimal in terms of control. In fact, smoothness is normally used as an indicator of skillfulness and well-trained motor performance [17]. However, a smooth control of the rocket in this task is also more difficult in practice, as the ability to quickly correct for trajectory errors is hampered, particularly when performed fast. Careful planning and dexterous operation are thus essential to the success of this strategy.

On the other hand, the 'intermittent' way is more manageable, and trajectory can be kept accurate by pressing in short bursts that quickly return the rocket to its path. It is, however, overall more demanding as the triggers are activated more. During this study, participant A mentioned to be tired of pressing the trigger after finishing the experiment, and together with participant $\mathrm{D}$ declared the triggers were noisy as they were pressing them a lot and very often. In this way, it could be argued that the 'intermittent' behaviour is not likely to be chosen by participants who believe can minimize their error using a less demanding 'continuous' strategy. It could therefore be hypothesized that participants with the 'intermittent' strategy could have adopted the 'continuous' one with more practice time, minimizing also their effort as they became more familiar with the task and improved their motor planning.

Spending more or less time in the task seems therefore to affect the relationship between error and smoothness. It should be highlighted how player J, who obtained the lowest error, took the longest time to complete the trials. This player was also the only one declaring to be an "expert" in manipulating Xbox 360 controllers (see Table I). In addition Fig. 5, seems to indicate that subjects found it difficult to achieve a low error with a smooth input. In the questionnaire, participants were asked to evaluate the difficulty of the task with a number from 1 to 10. Participants $\mathrm{G}, \mathrm{H}$ and $\mathrm{I}$ chose 3, 5 and 7, respectively. While $\mathrm{B}, \mathrm{C}$ and $\mathrm{E}$ chose 7,7 and 8, this could indicate that participants who put more time in the task did so consciously as they perceived the task as more complicated, however additional subjects would be needed to confirm this hypothesis.

In order to asses how motor behaviour varied along trials and how was this related to performance improvement, smoothness and time evolution was studied in every player. However, a clear tendency was not found for the performance time and a change in the input profile along trials was only observed for participant D. This player, as shown in Fig. 6, seemed to start with a "continuous" motor behaviour and transfer to the 'intermittent' one during the second trial, as it is indicated by the change in the smoothness value. This change in behaviour seems to entail a tracking improvement, 

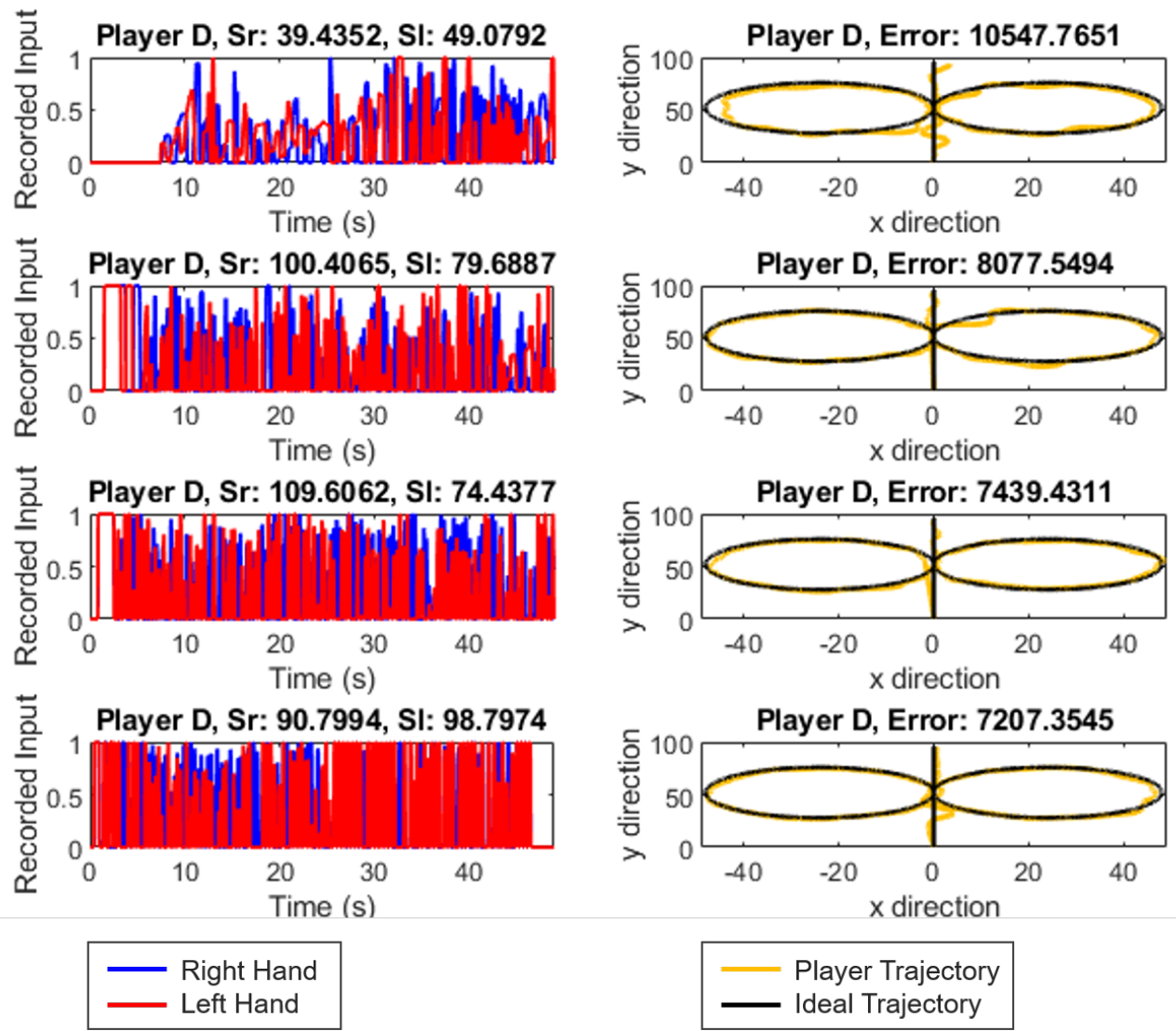

Player Trajectory

Ideal Trajectory

Fig. 6. Evolution along trials for player D. The figure shows the right hand and left hand inputs (Left), and corresponding output trajectories (Right) for trials 1, 2, 3 and 10 of player D. Both smoothness (Sl for left input and Sr for right input) and error are displayed for each trial.

as showed by the decrease in the error. However, with the recorded data no further assumptions on the motor learning can be made. In a longer study, changes in behaviour could be further studied and compared across participants. It would be interesting to analyze if participants with a "intermittent" behaviour change to a 'continuous' one as trials evolve, to minimize effort once task dexterity is achieved. This would confirm the hypothesis that motor behaviour depends on how well participants can minimize error. It would also be interesting to explore if improvement along trials correlates with changes in performance time.

\section{CONCLUSION AND FUture WORK}

This exploratory study found that two different motor behaviours arose when participants were tested on a redundant bimanual task. Further experimentation, with a larger number of participants and a higher number of repetitions, will be required to discover the leading causes of this behaviours. For the moment, it can be hypothesized that the "intermittent" behaviour might convey more effort but might facilitate error minimization, being ideal for participants who prefer to minimize their tracking error and are novel in the environment. It can be also concluded that in general participants were aware of their choices, which might be due to the specific demographics as all participants were students in the gaming field.

In order to be able to characterize and identify the causes of the different behaviours, future work will investigate the use of a bimanual control model of this task. One option would be to use a game-theory based controller [19], [20] to model the human motor control during this task. A game theory-based framework would allow the consideration that the control of each hand is dependent on the control of the other, while each hand tries to minimize a certain cost-function related to both the other hand's and its own error and effort.

The results presented in this paper are important for development new video game models [14], control interfaces for robots [21] and for understanding bimanual coordination to novel neuromotor rehabilitation technologies [22].

\section{APPENDIX: MODEL PARAMETERS}

The parameters $M_{r}, M_{l}, N_{r}, N_{l}, P_{r}$ and $P_{l}$ refer to dynamic properties of the vehicle model and were defined as described in [16], [23]. The values used in this work for the parameters below can be found in Table II. The following equations were used to calculate the model parameters.

$$
M_{r}=\frac{1}{\gamma_{r}}\left(\gamma_{r}^{2} J m_{r}+J w_{r}+\frac{R_{r}^{2}}{2}\left(\frac{M}{2}+\frac{J}{T^{2}}\right)\right),
$$




$$
\begin{gathered}
M_{l}=\frac{1}{\gamma_{l}}\left(\gamma_{l}^{2} J m_{l}+J w_{l}+\frac{R_{l}^{2}}{2}\left(\frac{M}{2}+\frac{J}{T^{2}}\right)\right), \\
N_{r}=\frac{1}{\gamma_{r}} \frac{R_{r} R_{l}}{2}\left(\frac{M}{2}-\frac{J}{T^{2}}\right), \quad N_{l}=\frac{1}{\gamma_{l}} \frac{R_{r} R_{l}}{2}\left(\frac{M}{2}-\frac{J}{T^{2}}\right), \\
P_{r}=\frac{1}{\gamma_{r}} \delta_{r}, \quad P_{l}=\frac{1}{\gamma_{l}} \delta_{l}
\end{gathered}
$$

TABLE II

VEHICLE CHARACTERISTICS.

\begin{tabular}{|c|c|c|}
\hline Parameter & Symbol & Value \\
\hline Gear ratio & $\gamma_{r}, \gamma_{l}$ & 40 \\
\hline Inertia of motor axis & $J_{m r}, J_{m l}$ & $10^{-} 8 \mathrm{~kg} \mathrm{~m}^{2}$ \\
\hline Inertia of wheel axis & $J_{r}, J_{l}$ & $10^{-} 5 \mathrm{~kg} \mathrm{~m}^{2}$ \\
\hline Inertia of the vehicle & $J$ & $0.2 \mathrm{~kg} \mathrm{~m}^{2}$ \\
\hline Wheel radius & $R_{r}, R_{l}$ & $0.05 \mathrm{~m}$ \\
\hline Mass of the vehicle & $M$ & $13 \mathrm{~kg}$ \\
\hline Tread of the vehicle & $T$ & $0.2 \mathrm{~m}$ \\
\hline Friction constant & $\delta_{r}, \delta_{l}$ & $0.15 \mathrm{~kg} \mathrm{~m}^{2} \mathrm{~s}^{-1}$ \\
\hline
\end{tabular}

\section{ACKNOWLEDGMENTS}

This research was supported by the EPSRC and the Centre for Intelligent Games and Game Intelligence (IGGI). I. Farkhatdinov was partially supported by EPSRC NCNR (EP/R02572X/1). The authors would like to thank J. Eden for his careful reading and comments on the manuscript.

\section{REFERENCES}

[1] C. E. Lang, J. R. MacDonald, D. S. Reisman, L. Boyd, T. Jacobson Kimberley, S. M. Schindler-Ivens, T. G. Hornby, S. A. Ross, and P. L. Scheets, "Observation of Amounts of Movement Practice Provided During Stroke Rehabilitation," Archives of Physical Medicine and Rehabilitation, vol. 90, no. 10, pp. 1692-1698, 2009.

[2] M. Maier, B. Rubio Ballester, E. Duarte, A. Duff, and P. F. M. J. Verschure, "Social Integration of Stroke Patients through the Multiplayer Rehabilitation Gaming System," pp. 100-114, Springer International Publishing, 2014.

[3] M. N. McDonnell, "Physical Activity Following Stroke," Archives of Physical Medicine and Rehabilitation, vol. 91, no. 4, pp. 665-666, 2010.

[4] G. Alankus, A. Lazar, M. May, and C. Kelleher, "Towards Customizable Games for Stroke Rehabilitation," in CHI 2010: Therapy and Rehabilitation, (Atlanta, Geogia (USA)), 2010.

[5] M. S. Cameirão, S. Bermúdez Badia, E. Duarte Oller, and P. Fmj Verschure, "The Rehabilitation Gaming System: a Review," Stud Health Technol Inform, vol. 145, no. 6, 2009.

[6] R. M. Proffitt, G. Alankus, C. L. Kelleher, and J. R. Engsberg, "Use of Computer Games as an Intervention for Stroke," Topics in Stroke Rehabilitation, vol. 18, pp. 417-427, 72011.

[7] M. King, J. Hijmans, M. Sampson, J. Satherley, and L. Hale, "Homebased stroke rehabilitation using computer gaming," New Zealand Journal of Physiotherapy, vol. 40, no. 3, pp. 128-134, 2012.

[8] S. P. Swinnen and N. Wenderoth, "Two hands, one brain: cognitive neuroscience of bimanual skill," TRENDS in Cognitive Sciences, vol. 8, no. 1, pp. 18-25, 2004.

[9] A. Wolf, R. Scheiderer, N. Napolitan, C. Belden, L. Shaub, and M. Whitford, "Efficacy and Task Structure of Bimanual Training Post Stroke: A Systematic Review," Topics in Stroke Rehabilitation, vol. 21, no. 3, pp. 181-196, 2014.

[10] S. P. Swinnen, "Intermanual coordination: From behavioural principles to neural-network interactions," Nature Reviews Neuroscience, vol. 3, no. 5, pp. 348-359, 2002.

[11] S. Hesse, G. Schulte-Tigges, M. Konrad, A. Bardeleben, and C. Werner, "Robot-assisted arm trainer for the passive and active practice of bilateral forearm and wrist movements in hemiparetic subjects," Archives of Physical Medicine and Rehabilitation, vol. 84, pp. 915-920, 62003.
[12] S. Kantak, S. Jax, and G. Wittenberg, "Bimanual coordination: A missing piece of arm rehabilitation after stroke," Restorative Neurology and Neuroscience, 2017.

[13] W.-W. Liao, J. Whitall, J. E. Barton, and S. M. Waller, "Neural motor control differs between bimanual common-goal vs. bimanual dual-goal tasks," Experimental Brain Research, vol. 236, pp. 1789-1800, 2018.

[14] M. Mace, N. Kinany, P. Rinne, A. Rayner, P. Bentley, and E. Burdet, "Balancing the playing field : Collaborative gaming for physical training," Journal of NeuroEngineering and Rehabilitation, vol. 14, no. 116, pp. 1-22, 2017.

[15] R. Oldfield, "The assessment and analysis of handedness: The Edinburgh inventory," Neuropsychologia, vol. 9, pp. 97-113, 31971.

[16] G. Dudek and M. Jenkin, "Differential Drive Kinematics," Computational Principles of Mobile Robotics, vol. 1, no. 3, pp. 1-5, 2010.

[17] S. Balasubramanian, A. Melendez-Calderon, A. Roby-Brami, and E. Burdet, "On the analysis of movement smoothness," Journal of NeuroEngineering and Rehabilitation, vol. 12, 2015.

[18] J. L. Emken, R. Benitez, A. Sideris, J. E. Bobrow, and D. J. Reinkensmeyer, "Motor Adaptation as a Greedy Optimization of Error and Effort," Journal of Neurophysiology, vol. 97, no. 6, pp. 3997-4006, 2007.

[19] Y. Li, G. Carboni, F. Gonzalez, D. Campolo, and E. Burdet, "Differential game theory for versatile physical humanrobot interaction," Nature Machine Intelligence, vol. 1, no. January, pp. 36-43, 2019.

[20] N. Jarrassé, T. Charalambous, E. Burdet, and M. O. Ernst, "A Framework to Describe, Analyze and Generate Interactive Motor Behaviors," PLOS ONE, vol. 7, no. 11, 2012.

[21] I. Farkhatdinov, J.-H. Ryu, and J. Poduraev, "A user study of command strategies for mobile robot teleoperation," Intelligent Service Robotics, vol. 2, no. 2, pp. 95-104, 2009.

[22] P. Rinne, M. Mace, T. Nakornchai, K. Zimmerman, S. Fayer, P. Sharma, J.-L. Liardon, E. Burdet, and P. Bentley, "Democratizing neurorehabilitation: How accessible are low-cost mobile-gaming technologies for selfrehabilitation of arm disability in stroke?," PloS one, vol. 11, no. 10, p. e0163413, 2016.

[23] S. Iida and S. Yuta, "Control of vehicle with power wheeled steering using feedforward dynamics compensation," in Proceedings IECON '91: 1991 International Conference on Industrial Electronics, Control and Instrumentation, pp. 2264-2269, IEEE. 